\title{
RASTREANDO CORPOS, PRODUZINDO SEXOS: A INSERÇÃO DA HIPERPLASIA ADRENAL CONGÊNITA NO TESTE DO PEZINHO $^{1}$
}

\author{
Janaína Freitas ${ }^{2}$ \\ Paula Sandrine Machado ${ }^{3}$
}

\begin{abstract}
RESUMO
0 presente artigo busca refletir sobre 0 modo pelo qual as intersexualidades estão sendo produzidas e manejadas em um contexto em que as novas biotecnologias constituem ferramentas cruciais no processo de remodelação dos corpos e de produção de subjetividades. Para tanto, a partir de uma "etnografia de arquivo", são analisados artigos científicos nacionais oriundos da área biomédica que abordam a triagem neonatal da Hiperplasia Adrenal Congênita (HAC), citada na literatura médica como a causa mais recorrente de intersexualidade, e sua inserção no Programa de Triagem Neonatal (Teste do Pezinho) no Brasil. Os resultados até então obtidos apontam para uma série de controvérsias em relação à inserção dessa condição nos programas de triagem, tais como um elevado número de casos "falso-positivos" e 0
\end{abstract}

\footnotetext{
${ }^{1}$ Este trabalho foi apresentado na V Reunião de Antropologia da Ciência e da Tecnologia (REACT), no Seminário Temático "Corporalidades, Saberes e Tecnologias", que ocorreu em maio de 2015, em Porto Alegre - RS. Agradecemos as contribuições dos e das participantes do ST, em especial as questões trazidas pelo professor Luís Fernando Sacchi dos Santos. 0 texto integra o trabalho de conclusão de curso em Ciências Sociais de Janaína Freitas, defendido na Universidade Federal do Rio Grande do Sul, em 2014, sob orientação da professora Paula Sandrine Machado.

${ }^{2}$ Mestranda em Antropologia Social, no Programa de Pós-Graduação em Antropologia Social da Universidade Federal do Rio Grande do Sul, Brasil. janaina.invernisi@gmail.com

${ }^{3}$ Professora do Programa de Pós-Graduação em Psicologia Social e Institucional, e do Programa de Pós-Graduação em Antropologia Social da Universidade Federal do Rio Grande do Sul, Brasil. machadops@gmail.com
} 
fato de a mesma não representar necessariamente um risco à vida dos sujeitos (um dos critérios importantes para inclusão nas triagens).

Palavras- chave: Intersexualidade. Hiperplasia Adrenal Congênita. Gênero. Ciência.

\title{
TRACKING BODIES, PRODUCING SEXES: THE INSERTION OF CONGENITAL ADRENAL HYPERPLASIA ON NEWBORN SCREENING
}

\begin{abstract}
The present article seeks to discuss the way through which intersexualities are being produced and managed in a context where new biotechnologies constitute themselves as crucial tools on the process of remodeling bodies and producing subjectivities. In order to achieve this, from an "ethnography of archives", national scientific articles that handle the insertion of Congenital Adrenal Hyperplasia (CAH) - which appears in medical literature as the most current cause of intersexuality in the Brazilian Newborn Screening Program - are analyzed. The results so far obtained point to a series of controversies related to this condition in newborn screenings, such as the elevated number of "false-positives" and the fact that it does not necessarily represent a risk to the subjects' lives (one of the most important criteria for the inclusion in screenings).
\end{abstract}

Keywords: Intersexuality. Congenital Adrenal Hyperplasia. Gender. Science.

\section{INTRODUÇÃO}

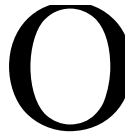

presente artigo visa refletir sobre a questão do "gerenciamento sociomédico das intersexualidades" (CABRAL, 2006; MACHADO, 2008) em um contexto de biomolecularização da sociedade (ROSE, 2011), no qual, cada vez mais intensamente, as biotecnologias são ferramentas cruciais no processo de remodelação dos corpos e de produção de subjetividades. Busca-se, mais especificamente, refletir sobre a forma pela qual os saberes biomédicos e especialmente as novas tecnologias diagnósticas ocidentais "performam" (MOL,

\footnotetext{
${ }^{4}$ Esse termo se refere ao conceito enact, proposto por Annemarie Mol (2003). Ele pode ser traduzido como atuar, decretar ou ainda performar. Entretanto, não há um consenso na tradução empregada, uma vez que a autora adverte que ele possui um significado "tão novo quanto possível” (MOL, 2003, p.41) daquele já empregado nas Ciências Humanas e Sociais. Nesse sentido, segundo ela, o uso deste termo parte do pressuposto que a existência dos objetos está absolutamente atrelada às práticas que o produzem. No caso do presente artigo, entendemos que a
} 
2007, p. 6) as intersexualidades e, mais amplamente, as diferenças corporais e as normatizações de gênero na contemporaneidade.

De acordo com Alice Dreger (1998), o termo intersexualidade surgiu no contexto médico, tendo sido cunhado no começo do século XX pelo biólogo Richard Goldschmidt, para designar corpos que se situavam entre o que era considerado como masculino e feminino. A temática da intersexualidade foi, por muito tempo (e ainda segue sendo), relegada às abordagens das ciências biomédicas 5. Contudo, a partir dos anos 1990, junto à emergência do movimento político intersex, a intersexualidade passou a ser tema de pesquisa das ciências humanas, principalmente no âmbito dos feminist studies (DREGER \& HERNDON, 2009).

Nos anos 1990, algumas das pessoas que integraram as primeiras gerações submetidas às diretrizes do protocolo de tratamento de John Money ${ }^{6}$ - primeiro médico a indicar as intervenções cirúrgicas em crianças intersexuais começaram a formar organizações e grupos de militância política intersex, sendo o pioneiro a Intersex Society of North America (ISNA). Estes grupos tinham como objetivo o compartilhamento de experiências e, em sua grande maioria, faziam a crítica aos tratamentos médicos não consentidos, lutando pelo fim das cirurgias precoces "corretoras" das genitálias que não se enquadravam no modelo do dimorfismo sexual (MACHAD0, 2008; KARKAZIS, 2008).

0 termo "intersexualidade", utilizado primeiramente no meio médico, passa a ser, então, reapropriado no âmbito da militância política, e utilizado a partir da ideia de "variação", no que se refere a um padrão binário - masculino e feminino - estabelecido culturalmente (CABRAL \& BENZUR, 2005, p. 283). Neste sentido, a opção por utilizar o termo "intersexualidade", neste artigo, remete à sua inscrição a um campo de estudos críticos sobre o tema nas ciências humanas e sociais, mas, sobretudo, situa-o nessa discussão política, que indica um afastamento da perspectiva patologizante biomédica, a qual pressupõe a necessidade de intervenções "corretoras" e não reconhece as diferenças nos

HAC depende das tecnologias e dos discursos, tais como aqueles empregados no Teste do Pezinho, para existir.

${ }^{5}$ De acordo com Mauro Cabral (2009), considerar modos não-medicalizados de abordar a intersexualidade segue sendo um dos desafios teóricos e políticos do campo.

${ }^{6}$ Esse psico-endocrinologista ficou conhecido por desenvolver, junto a outros pesquisadores, a teoria do desenvolvimento da "identidade de gênero". Esta teoria estabelecia uma diferenciação entre o sexo biológico e o sexo psíquico. Nesse sentido, em casos de crianças intersex, segundo ele, era possível adequar o sexo biológico e a identidade de gênero através de cirurgias e tratamentos farmacológicos (HARAWAY, 2004, p. 217; MACHADO, 2008, p. 192). 
corpos como possíveis e desejáveis. A partir dessa perspectiva, propomo-nos analisar, mais especificamente, uma questão atual no contexto do gerenciamento sociomédico da intersexualidade: a inserção da Hiperplasia Adrenal Congênita (HAC) no Programa de Triagem Neonatal no Brasil.

Desde 2012, a chamada Hiperplasia Adrenal Congênita (HAC), que aparece na literatura médica como a causa mais recorrente de intersexualidade (JESUS \& BASTOS, 2004), foi incluída no rol de condições diagnosticadas pelo Programa de Triagem Neonatal (Teste do Pezinho) no Brasil. De acordo com Barra et al. (2012), a triagem para essa condição não é aceita de maneira consensual mesmo dentro do contexto biomédico, uma vez que sua inclusão no programa de triagem gera uma série de polêmicas e debates.

Neste trabalho, realizamos uma pesquisa de orientação etnográfica, tendo como referência a "etnografia do arquivo", discutida pela antropóloga Olívia Gomes da Cunha (2004, p. 287). No presente caso, os "arquivos" são constituídos majoritariamente de artigos científicos nacionais oriundos da área biomédica, que abordam especificamente a inserção da HAC no programa de triagem neonatal no Brasil. Além destes textos, utilizamos como material empírico os cartazes de divulgação do Teste do Pezinho - respectivos aos anos de 2012, 2013 e 2014 - e outros dois artigos médicos que tratam mais especificamente do protocolo de tratamento da HAC. Estes outros documentos foram utilizados por se mostrarem importantes, ao longo da pesquisa, para compreender 0 manejo médico atual desta condição.

A opção por etnografar esses textos médicos se deu em função das justificativas para inserção dessa condição no programa de triagem, encontradas no texto da Portaria $\mathrm{n}^{0} 2.829$ de 14 de Dezembro de 2012, documento administrativo, emitido pelo Ministério da Saúde, que recomendou a inclusão do diagnóstico para Hiperplasia Adrenal Congênita e Deficiência de Biotidinase ${ }^{7}$ no Programa Nacional de Triagem Neonatal. De acordo com o texto da Portaria, a inclusão da triagem para essas condições é justificada pelos seguintes critérios:

Considerando a necessidade de ampliar 0 acesso à triagem neonatal para detecção de outras doenças e os estudos científicos nacionais $e$

\footnotetext{
${ }^{7}$ Condição incluída no Teste do Pezinho, junto à HAC. Segundo as descrições médicas, trata-se de uma doença genética caracterizada por uma falha parcial ou total da biotina, uma das vitaminas que fazem parte do Complexo B. Disponível em:

$<$ http://www.medicina.ufmg.br/nupad/triagem/triagem_neonatal_deficiencia_de_biotinidase_co nceito.html >. Acesso em: 05/07/2014.
} 
internacionais recomendando a triagem neonatal em caráter universal para hiperplasia adrenal congênita e a deficiência de biotinidase; Considerando estudos nacionais justificando a importância epidemiológica e a avaliação do custo-benefício para o tratamento precoce da hiperplasia adrenal congênita $e$ a deficiência de biotinidase. (MINISTÉRIO DA SAÚDE, 2012: 54, grifo nosso).

Conforme mencionado no texto grifado da Portaria, a inserção da HAC foi balizada por "estudos científicos nacionais e internacionais". Entretanto, este documento não citava especificamente os artigos científicos aos quais se referia. Desse modo, foi feita uma busca dos artigos que tratavam da triagem neonatal dessa condição. Os artigos científicos foram buscados através do Portal de Periódicos da Capes e do PubMed, portal de periódicos médicos estadunidense. As palavras-chave utilizadas na busca foram: "triagem neonatal para hiperplasia adrenal congênita", bem como a sua versão em língua inglesa: "newborn screening for congenital adrenal hyperplasia".

A partir dessas buscas, foram encontradas 208 publicações - todavia, apenas 103 estavam disponíveis gratuitamente para download - dentre essas, apenas oito eram brasileiras. Assim, foi priorizada, nessa etapa da pesquisa, a análise dos artigos científicos nacionais que tratavam da triagem para HAC, uma vez que 0 trabalho objetivava estudar o caso brasileiro e mapear essas produções e suas características, vinculações institucionais, grupos de pesquisa e profissionais envolvidos no país.

Dos oito estudos nacionais encontrados, um se baseava em uma tese de doutorado em Endocrinologia, da Universidade Federal do Rio Grande do Sul (composta por três artigos publicados em parceria com outros pesquisadores); outro se baseava em uma dissertação de mestrado em Ciências da Saúde, da Universidade Federal de Minas Gerais; três artigos foram publicados nos "Arquivos Brasileiros de Endocrinologia e Metabologia"; um no "Jornal de Pediatria"; um na "Revista da Associação Médica Brasileira", e outro na "Revista Brasileira em Promoção da Saúde (RBPS)". Sendo assim, os oito artigos analisados se referem à totalidade de publicações encontradas que obedeciam aos critérios préestabelecidos supracitados.

Fizemos o esforço de mapear os principais argumentos, saberes e justificativas acerca da inserção da HAC no teste. Assim, a pesquisa acabou desvelando questões relacionadas ao manejo médico desta condição, isto é, que se referem a algumas possibilidades diagnósticas, às terapêuticas possíveis, e 
também às controvérsias acerca da triagem da HAC, sinalizando, assim, para os modos através dos quais esta condição estava sendo "performada" (MOL, 2003) por essa nova técnica diagnóstica. Deste modo, optamos por dividir o presente artigo em quatro partes. A primeira traz uma breve descrição dos artigos científicos analisados. A segunda trata da forma pela qual a HAC e seu manejo atual são descritos no material analisado. A terceira parte discute a triagem neonatal, de uma maneira mais ampla, e, mais particularmente, a triagem neonatal para HAC. Na última seção, apresentamos algumas das controvérsias, no que tange à triagem da HAC, mapeadas ao longo da pesquisa.

\section{OS ARTIGOS CIENTÍFICOS SOBRE A TRIAGEM NEONATAL DA HAC}

Nesta seção, faremos uma breve descrição dos artigos científicos analisados, a qual contempla a estrutura dos artigos, bem como os locais onde foram produzidos e posteriormente publicados. Os textos encontrados são majoritariamente oriundos das áreas da Endocrinologia e da Pediatria, contudo, alguns deles foram produzidos por pesquisadores da Genética e também da Biologia Molecular. As publicações ocorreram em revistas tais como os "Arquivos Brasileiros de Endocrinologia e Metabologia" e o "Jornal de Pediatria", principais periódicos brasileiros nessas especialidades. Tal fato permite visualizar quais são os saberes que mais recorrentemente abordam a questão da HAC - especialmente no que se refere à triagem - e que, nesse sentido, são mais "legitimados" a "falar" sobre esta condição.

Os artigos encontrados são bastante recentes, datando do período entre 2005 e 2014. Ademais, todos obedecem a uma estrutura própria de artigos acadêmicos biomédicos, apresentando os objetivos, a metodologia e os resultados. No que tange ao seu conteúdo, trata-se de textos sucintos, compostos, no máximo, por oito páginas, escritos em parceria com outros pesquisadores, com exceção da dissertação de mestrado de Isabela Pezzuti, que também compõe os documentos analisados. Cinco textos foram escritos em língua portuguesa e três deles (incluindo os artigos que compõe a tese de doutorado de Elizabeth Silveira), embora tenham sido publicados em periódicos nacionais, foram escritos em língua inglesa. 0 quadro abaixo permite visualizar os estudos encontrados: 
Quadro 1 - Síntese dos artigos analisados sobre a triagem neonatal da Hiperplasia Adrenal Congênita

\begin{tabular}{|c|c|c|}
\hline Título Artigo & Autoria & Publicado em/Ano \\
\hline $\begin{array}{c}\text { Triagem Neonatal para } \\
\text { Hiperplasia Adrenal Congênita }\end{array}$ & $\begin{array}{c}\text { Cristina Botelho Barra, Ivani Novato } \\
\text { Silva, Isabela Pezzuti e José Nélio } \\
\text { Januário }\end{array}$ & $\begin{array}{l}\text { Revista Associação } \\
\text { Médica Brasileira } \\
\text { (2012) }\end{array}$ \\
\hline $\begin{array}{l}\text { Hiperplasia adrenal congênita no } \\
\text { Brasil: incidência, custos da } \\
\text { triagem neonatal e aplicação } \\
\text { clínica da biologia molecular } \\
\text { (Tese de Doutorado que se } \\
\text { constitui em três artigos) }\end{array}$ & $\begin{array}{l}\text { Elizabeth Silveira Lemos (escreve os } \\
\text { três artigos que compõe a tese em } \\
\text { coautoria com outros pesquisadores } \\
\text { como Regina Elnecave, Tânia } \\
\text { Bachega, Eliane dos Santos, Ivana } \\
\text { Nader, Berenice Mendonça, Jorge } \\
\text { Gross, Suzi Camey, Isaias Prestes, } \\
\text { Vivian Moura e Emilia Pinto) }\end{array}$ & $\begin{array}{l}\text { Tese de Doutorado } \\
\text { Programa de Pós- } \\
\text { Graduação em } \\
\text { Ciências Médicas: } \\
\text { Endocrinologia } \\
\text { (2008) }\end{array}$ \\
\hline $\begin{array}{c}\text { Triagem neonatal para } \\
\text { hiperplasia adrenal congênita: } \\
\text { experiência do estado do Rio de } \\
\text { Janeiro }\end{array}$ & $\begin{array}{l}\text { Cláudia Cardoso, Armando Fonseca, } \\
\text { Maria de Fátima Oliveira, Basílio } \\
\text { Pereira, Marília Guimarães }\end{array}$ & $\begin{array}{l}\text { Arquivos Brasileiros de } \\
\text { Endocrinologia e } \\
\text { Metabologia } \\
\text { (2005) }\end{array}$ \\
\hline $\begin{array}{l}\text { Avaliação do programa-piloto de } \\
\text { triagem neonatal para } \\
\text { hiperplasia adrenal congênita no } \\
\text { estado de Minas Gerais }\end{array}$ & Isabella Leite Pezzuti & $\begin{array}{c}\text { Dissertação de } \\
\text { Mestrado (UFMG) } \\
(2010)\end{array}$ \\
\hline $\begin{array}{c}\text { Triagem Neonatal para } \\
\text { Hiperplasia Adrenal Congênita: } \\
\text { Considerações Sobre a Elevação } \\
\text { Transitória da 17- } \\
\text { hidroxiprogesterona. } \\
\end{array}$ & $\begin{array}{c}\text { Crésio Alvez, } \\
\text { Valdi Balesteri Júnior e Maria Betânia } \\
\text { Pereira Toralles }\end{array}$ & $\begin{array}{l}\text { Revista Brasileira em } \\
\text { Promoção da Saúde } \\
\text { (2006) }\end{array}$ \\
\hline $\begin{array}{l}\text { A three-year follow-up of } \\
\text { congenital adrenal hyperplasia } \\
\text { newborn screening }\end{array}$ & $\begin{array}{c}\text { Isabela L. Pezzuti, Cristina B. Barra, } \\
\text { Rafael M. Mantovani, José N. Januário } \\
\text { e Ivani N. Silva }\end{array}$ & $\begin{array}{l}\text { Jornal de Pediatria } \\
\text { (2014) }\end{array}$ \\
\hline $\begin{array}{l}\text { Weight-adjusted neonatal } 170 \mathrm{H}- \\
\text { progesterone cutoff levels } \\
\text { improve the efficiency of } \\
\text { newborn screening for congenital } \\
\text { adrenal hyperplasia. }\end{array}$ & $\begin{array}{l}\text { Giselle Hayashi, Cláudia Faure, Maria } \\
\text { Fernanda Brondi, Carla Vallejos, } \\
\text { Daiana Soares, Érica Oliveira, Vinícius } \\
\text { N. Brito, Berenice B. Mendonca, } \\
\text { Tânia A. S. S. Bachega }\end{array}$ & $\begin{array}{l}\text { Arquivos Brasileiros de } \\
\text { Endocrinologia e } \\
\text { Metabologia } \\
\text { (2011) }\end{array}$ \\
\hline $\begin{array}{l}\text { Heterozygosis for CYP21A2 } \\
\text { Mutation Considered as 21- } \\
\text { Hydroxylase Deficiency in } \\
\text { Neonatal Screening }\end{array}$ & $\begin{array}{l}\text { Sofia Marini Soardi, Fernanda Coeli, } \\
\text { Víctor Maturana, Márcia da Silva, } \\
\text { Renan Bernardi, Giselle Justo e } \\
\text { Maricilda de Mello }\end{array}$ & $\begin{array}{c}\text { Arquivos Brasileiros de } \\
\text { Endocrinologia e } \\
\text { Metabologia } \\
\text { (2008) }\end{array}$ \\
\hline
\end{tabular}

Fonte: Elaboração própria. 
É importante ressaltar que, dentre os oito artigos analisados, três deles foram escritos quase pelos mesmos autores e se referem à apresentação e discussão de um caso específico: 0 projeto-piloto empreendido no estado de Minas Gerais, entre os anos de 2007 e 2008. Este projeto piloto teve como objetivo contribuir para o "estabelecimento de um programa de rotina" no estado (BARRA et al, 2014, p.302) e foi incluído no Programa de Triagem Neonatal de Minas Gerais. Embora não iremos nos deter a esse ponto no presente artigo, cabe mencionar que o programa de triagem de Minas Gerais foi criado em parceria com 0 NUPAD (Núcleo de Ações e Pesquisas em Apoio Diagnóstico) da Universidade Federal de Minas Gerais, ao qual alguns dos pesquisadores acima mencionados estão vinculados. Ainda, a partir da análise dessas vinculações institucionais e pessoais, ficou explícito que alguns pesquisadores envolvidos nas publicações resultantes do projeto piloto, tais como Isabella Pezzuti, Cristina Barra, e Ivani Novato Silva, do estado de Minas Gerais, aparecem também em parte considerável dos outros artigos encontrados, apontando para a formação de uma rede pequena de atores que abordam mais intensamente a temática no país.

\section{A HIPERPLASIA ADRENAL CONGÊNITA NO DISCURSO BIOMÉDICO}

Nos artigos científicos analisados, buscou-se as definições que estes traziam da HAC e o seu manejo médico, a fim de compreender como essa condição era performada (MOL, 2003) pelo discurso biomédico. A totalidade dos textos utiliza, basicamente, as mesmas definições de HAC. Afirmam que a Hiperplasia Adrenal Congênita (HAC), também chamada Hiperplasia Suprarrenal Congênita (HSRC), pode ser caracterizada como uma gama de condições genéticas que envolvem as glândulas adrenais, responsáveis pela produção dos "hormônios cortisol, aldoresterona e androgênios", (PEZZUTI et al., 2014; SILVEIRA, 2008) os quais ajudariam a regular algumas funções do organismo, tais como o metabolismo de minerais (no caso da aldosterona) e o metabolismo de carboidratos, proteínas e gorduras (no caso do cortisol).

Segundo os/as autores/as, a HAC pode se apresentar clinicamente de várias formas, que dependem de uma suposta "gravidade" da "deficiência enzimática", geralmente dividida em duas formas principais: a "forma clássica" e a "forma não clássica ou tardia" (ALVEZ, 2006, p. 204; PEZZUTI, 2010, p. 19-20). A primeira, que geralmente provoca algumas manifestações clínicas desde 0 período neonatal, é ainda subdividida em "forma perdedora de sal", que corresponderia a 75\% dos casos, e "forma virilizante simples", relacionada a $25 \%$ dos casos. 
A respeito das apresentações clínicas da $\mathrm{HAC}$, os artigos analisados afirmam que "mulheres" que apresentam a forma clássica da doença nascem com o que, em termos biomédicos, chama-se "genitália ambígua". Ainda, segundo estes dados, $75 \%$ das pessoas com a forma clássica nascem com "perda de sal" condição que, se não tratada a tempo, pode levar à morte. Os artigos médicos também afirmam que indivíduos com a "forma não clássica ou tardia da doença" podem ser "assintomáticos" ou apresentar sinais de "hiperandrogenismo" mais tardiamente na infância ou na vida adulta. 0 "hiperandrogenismo" é caracterizado, na linguagem médica, como um distúrbio endocrinológico que poderia ter como manifestações clínicas principais o excesso de pelos, acne, queda de cabelo, aumento da libido, virilização, puberdade precoce e ciclos menstruais irregulares (YARAK, 2005, p. 396).

De acordo com um dos artigos sobre o tratamento da HAC analisados, escrito por Tânia Bachega (2001), o tratamento, que deve ser mantido pelo resto da vida, pode ocorrer tanto no período pré-natal, com o uso de uma substância chamada "dexametasona"; como no período neonatal, na infância ou ainda no período pós-puberal, através de intervenções cirúrgicas e farmacológicas que incluem o uso de uma série de medicamentos, como os "corticóides", e cirurgias que visam à redução do clitóris nos sujeitos assignados como meninas no nascimento.

Segundo Bachega, o tratamento pré-natal, feito com 0 uso de "dexametasona", teria como maior objetivo "evitar a virilização da genitália externa de fetos femininos afetados" (BACHEGA, 2001, p. 65). Conforme apontam Dreger et al. (2012), em um artigo em que fazem algumas críticas ao uso da dexametasona, 0 tratamento com essa substância é controverso, uma vez que não há estudos longitudinais acerca das consequências de sua utilização. No entanto, alguns médicos seguem reconhecendo e indicando o seu uso como terapêutica pré-natal. Nesse sentido, embora quase não existam estudos longitudinais sobre 0 uso dessa substância, Dreger et al. (2012) argumentam que um pequeno estudo, realizado em 1971, aponta para casos de "adrenocarcinoma vaginal", bem como de "problemas no sistema cardiovascular", em indivíduos expostos à "dexametasona", tanto no que se refere às mães quanto aos fetos.

No período neonatal, a terapêutica para os casos que podem gerar uma "crise de perda de sal" (que, conforme mencionado, se não tratada a tempo, leva à morte) é realizada com uma substancia conhecida como "hidrocortisona", e através da "reposição de sódio" (BACHEGA et al., 2001, p. 67). 0 tratamento pósnatal em crianças com a forma "virilizante simples", isto é, sem "perda de sal" 
também, geralmente, é feito com "hidrocortisona" (BACHEGA et al., 2001, p. 67). Ademais, nas meninas é realizada uma "cirurgia corretora da genitália externa" que consiste em uma "clitoroplastia", isto é, uma redução cirúrgica do clitóris. Estas intervenções cirúrgicas são realizadas, geralmente, até os dois anos de idade, em função do argumento biomédico que considera que "a identidade de gênero" de uma criança se consolidaria durante esse período. Tal argumento é baseado no protocolo de John Money, em sua "versão interacionista" da teoria sobre identidade de gênero (HARAWAY, 2004, p. 217), além de ter sido muito informado por algumas teorias psicológicas e psicanalíticas referentes ao desenvolvimento psicossexual.

Segundo as prescrições encontradas no artigo de Tânia Bachega (2001), a "forma não-clássica" de HAC deveria ser tratada apenas quando (e se) fosse "sintomática". Todavia, vale ressaltar que os sintomas descritos são bastante inespecíficos, tais como uma quantidade de pelos considerada inadequada, a acne, os ciclos menstruais irregulares e até mesmo a calvície. Nesse sentido, situações que podem ser recorrentes na vida de qualquer sujeito, como as manifestações descritas acima, são, no presente caso, entendidas como sinais de uma possível enfermidade.

No seguinte trecho desse mesmo artigo de Bachega et al. (2001, p. 70), que aborda a "função gonadal" de seus "pacientes", eles constatam primeiramente que 18 das 27 mulheres adultas com a forma clássica referiram ter uma "atividade sexual satisfatória". Entretanto, na sequência do parágrafo, afirma-se que, antes do início da atividade sexual, essas mulheres foram submetidas a intervenções cirúrgicas e/ou ao uso de dilatadores vaginais, fato que expressa que a heterossexualidade é utilizada como balizador no discurso e na prática médica (MACHAD0, 2008; CABRAL, 2006), bem como critério avaliativo do que se considera uma "atividade sexual satisfatória". Ainda, conforme visto também no artigo de Bachega et. al. (2001, p. 69) a regularidade do ciclo menstrual e a possibilidade de engravidar são igualmente entendidas como aspectos que possibilitam avaliar a eficácia do tratamento da HAC.

Nos relatos dos resultados da "avaliação psicológica", os autores (BACHEGA et al. 2001) afirmam que as "pacientes" que não foram tratadas na infância apresentaram identificação com o "sexo social masculino". Além disso, utilizam os termos "libido feminina", "libido masculina" e "atividade sexual adequada" (BACHEGA et al., 2001, p. 70), deixando evidente que o desejo heterossexual é entendido como mais "adequado", segundo os critérios avaliados. Assim, demonstram novamente a "heteronormatividade" (BUTLER, 2003) presente nos 
critérios por eles utilizados para a dita avaliação psicológica de indivíduos com HAC.

No final do artigo, os/as autores/as afirmam que uma série de "novos tratamentos" tem sido proposta. Dentre eles, há uma possibilidade caracterizada como "futura" que eles denominam de "injeção intra-adrenal de adenovírus não replicante contendo a sequência genômica do CYP21 humano corrigida" (BACHEGA et al., 2001, p. 70). Isto é, diz respeito a um tratamento, baseado em terapia gênica, no qual se utilizam técnicas da biologia molecular, com o propósito de "prevenir" ou "curar" a HAC, através de uma correção na suposta falha contida em um dos cromossomos descritos como causadores da HAC. Nesse sentido, tanto o uso precoce de "dexametasona", quanto o tratamento com "terapia gênica" explicitam a forma pela qual novas tecnologias, muitas delas baseadas na genética e na biologia molecular, investem esforços no sentido de controlar, cada vez mais precocemente, os corpos que não se enquadram no modelo do dimorfismo sexual.

Neste sentido, cabem aqui as reflexões de Marko Monteiro (2005) acerca da genética e da biologia molecular como saberes potencialmente eugênicos, uma vez que possibilitam a escolha de fetos com características específicas, bem como permitem modificar outras já existentes. Ainda, constatou-se, a partir deste material analisado e dos sentidos que nele se encontram atribuídos à $\mathrm{HAC}$, que a discussão e o manejo desta condição em um nível "molecular", quando ela é "performada" (MOL, 2003) como níveis de esteróides e mutações cromossômicas, acaba por distanciar a relação direta com o seu nível "molar", o da genitália considerada "ambígua" de acordo com os padrões biomédicos. Nesse movimento de distanciamento, a "preocupação social" com a "genitália ambígua", com vistas a uma suposta manutenção do modelo do dimorfismo sexual, dá lugar a um discurso de que as intervenções biomédicas estariam apenas "corrigindo", de maneira "não-invasiva", uma "natureza que se desviou do seu curso".

\section{RASTREANDO CORPOS: A TRIAGEM NEONATAL}

A triagem neonatal, desenvolvida no final da década de 1950, é um procedimento padrão na maioria dos países. Desde a década de 1960 é recomendada pela Organização Mundial de Saúde, constituindo-se como um

\footnotetext{
${ }^{8}$ Este termo faz referência aos chamados testes não-invasivos pré-natais que visam a diagnosticar possíveis enfermidades nos fetos, como a $\mathrm{HAC}$, através de exames realizados nas mães durante 0 período pré-natal.
} 
exame obrigatório, gratuito e oferecido pelo Estado. Conforme sugerido pelo material de divulgação do Teste do Pezinho, além de ser entendido como um dever da família, que é obrigada a submeter seus filhos ao teste, é caracterizado como uma obrigação especificamente da mãe. Frases contidas no material, como as citadas abaixo, são constituídas como "apelos" morais que se dirigem majoritariamente às "mães":
"Um Teste de Amor: Mamãe, obtenha aqui informações importantes sobre o teste do pezinho do seu bebê";
"Teste do Pezinho: Um direito de todo o recém-nascido. Um dever de todos os pais"; ou
"Teste do Pezinho: indispensável como o amor de mãe"; ou ainda, "Isso também é carinho: 0 Teste do Pezinho é a primeira prova de amor que você pode dar para o seu filho"”

Deste modo, pode-se afirmar que, no programa de triagem, estão em jogo, além da responsabilização do Estado e da família, uma expectativa de gênero que relaciona a mulher à conservação da saúde familiar e que a localiza como protagonista nesse projeto de saúde e de controle.

Atualmente, o Programa Nacional de Triagem Neonatal possui quatro fases de habilitação. A primeira visa diagnosticar condições conhecidas como "feniceltonúria" e "hipotireoidismo"; a segunda, além daquelas já mencionadas, inclui a detecção de "anemia falciforme e outras hemoglobinopatias"; a terceira inclui "fibrose cística"; e a fase IV é aquela que prevê o diagnóstico de "HAC", juntamente ao de "deficiência de biotidinase". Embora a Portaria da quarta fase tenha sido publicada em 2012, foi apenas em novembro de 2013 que o Estado do Rio Grande do Sul, através da Resolução No 591/13 - CIB/RS, juntamente com a Área Técnica da Saúde da Criança e do Adolescente da Secretaria Estadual de Saúde e o Serviço de Referência Estadual em Triagem Neonatal, decretou a passagem à fase IV. Somente no dia cinco de maio de 2014, o Hospital MaternoInfantil Presidente Vargas, de Porto Alegre, considerado um serviço de referência para Triagem Neonatal, passou a implantar a quarta fase do teste.

De acordo com Cardoso et al. (2005), a triagem neonatal para a Hiperplasia Adrenal Congênita foi possível a partir 1977, quando Pang e outros pesquisadores desenvolveram uma técnica de ensaio para a dosagem da 170H Progesterona

\footnotetext{
${ }^{9}$ Essas frases foram encontradas nos panfletos de divulgação do Teste do Pezinho respectivos aos anos de 2012, 2013 e 2014. Disponível em: < http://www.unisert.org.br/campanhas.html>
} 
(170HP), um esteroide produzido pelas glândulas adrenais, a partir da coleta de sangue de bebês recém-nascidos em papel filtro. 0 primeiro programa mundial de triagem para HAC foi implantado no Alasca, devido à alta incidência dessa condição entre os esquimós Yupik (CARDOSO et al. apud PANG et al., 2005). Em seguida, o teste foi introduzido em outros países. No Brasil, para uma condição passar a integrar 0 rol de doenças diagnosticadas pelo programa de triagem neonatal deve apresentar os seguintes critérios:

Para a inclusão de uma doença em um programa de triagem neonatal, alguns requisitos devem ser atendidos: as doenças consideradas apropriadas devem ser relativamente frequentes na população triada, apresentar morbidade e mortalidade significativas quando não tratadas precocemente e ter resposta benéfica ao tratamento. (BARRA et al., 2012, p. 460, grifo nosso)

Como aponta Silveira (2008, p. 1), na sua tese de doutorado, a triagem para HAC no Brasil, por ainda não estar incluída no programa de triagem naquela época, era realizada apenas em laboratórios privados ou dependia de incentivos municipais e/ou estaduais para ser disponibilizada gratuitamente. Nesse sentido, Pezzuti (2010) defende a inclusão dessa condição na triagem neonatal utilizando os seguintes argumentos:

A HAC por D21-0H é considerada doença elegível para ser incluída nos programas de triagem neonatal, por ser relativamente frequente, potencialmente fatal na infância e que pode ser triada por medida hormonal simples. Além disso, possui tratamento eficaz, simples e de baixo custo que, quando instituído precocemente, pode prevenir morbimortalidade. (PEZZUTI, 2010, p. 17)

Ainda, esta autora acrescenta que "os principais objetivos da triagem para HAC seriam: a) detectar os casos graves da doença (perdedores de sal) para prevenção de choque, dano cerebral e morte por meio de tratamento présintomático; b) impedir ou reduzir o tempo de registro civil incorreto que pode ocorrer em meninas afetadas virilizadas" (PEZZUTI, 2010, p.17). Entretanto, a inclusão da HAC na triagem neonatal não é consensual e "integra o grupo que gera polêmica para inclusão nesse programa de rastreamento" (BARRA et al., 2012, p. 462). 


\section{MAPEANDO CONTROVÉRSIAS ACERCA DA INSERÇÃO DA HAC NO TeSTE DO PEZINHO}

Como propõe Latour (2000, p. 39), fez-se o esforço de mapear as "questões de interesse", isto é, "os fatos em elaboração", os quais ainda não se tornaram rotineiros no contexto estudado. Durante uma breve conversa com uma médica envolvida na triagem neonatal em Porto Alegre, esta afirmou que julgava o tema de pesquisa "muito importante" ${ }^{\text {, }}$ uma vez que a inserção da HAC na triagem neonatal estava "dando muita dor de cabeça" para os profissionais envolvidos no programa. Segundo seu relato, isto estaria ocorrendo em função das "controvérsias" acerca da sua inclusão no programa de rastreamento, especialmente em relação à elevada taxa de resultados falso-positivos, questão com a qual já havíamos nos deparado na pesquisa com os artigos biomédicos. Nesse sentido, o termo controvérsia apareceu inicialmente como um termo "êmico", mas é abordado analiticamente nesse trabalho no sentido proposto por Latour (2012, p. 44), o qual entende as controvérsias sociotécnicas como "rastros" para se pensar as conexões sociais.

Nessa seção, apresentamos e discutimos algumas dessas controvérsias. Embora elas estejam absolutamente entrelaçadas umas às outras, para fins analíticos, estabelecemos uma divisão entre elas. A primeira, e talvez a mais mencionada, diz respeito ao elevado número de resultados falso-positivos, isto é, àqueles casos em que o resultado dos exames é positivo, mas o sujeito não possui a condição para a qual foi diagnosticado. Todos os artigos científicos analisados destacam essa questão e alguns deles propõem mudanças na abordagem diagnóstica que visem a reduzir essa taxa. Os textos afirmam que os resultados falso-positivos podem estar relacionados à prematuridade, ao uso de alguns tipos de medicamentos durante a gestação e mesmo ao estresse.

Uma das soluções para reduzir a alta taxa de resultados falso-positivos, afirmam alguns dos textos analisados, poderia ser a mudança no "ponto de corte" (BARRA et al., 2012, p. 463), isto é, nos valores - medidos a partir de "níveis séricos de 17-0HP” - utilizados para distinguir os sujeitos que possivelmente possuiriam essa condição, daqueles com taxas consideradas normais. Nesse sentido, Pezzuti (2010, p. 25-26) argumenta que esses "níveis séricos de 17-0HP" se apresentariam em valores significativamente diferentes nas distintas formas de HAC.

\footnotetext{
${ }^{10}$ Os termos entre aspas nesse parágrafo foram aqueles utilizados pela médica na ocasião em que apresentamos a proposta de pesquisa.
} 
Assim, a autora deixa evidente que, devido a essas diferenças nos níveis séricos de 17-0HP, haveria a possibilidade de diminuir as taxas de resultados falso-positivos ao se diagnosticar apenas os casos com "perda de sal". Nesse caso, deveria haver um "aumento" no "ponto de corte" para que apenas os casos com "perda de sal", isto é, aqueles que podem apresentar consequências graves à saúde, fossem diagnosticados. Todavia, conforme se argumenta nos textos, a opção por um aumento no "ponto de corte", como estratégia de redução dos casos falso-positivos, não permitiria a detecção dos casos "virilizantes simples".

Desse modo, embora muitos autores mencionem que o principal objetivo da triagem para HAC seria diagnosticar os casos de "perda de sal", uma vez que essa é a apresentação mais "grave e lesiva" da condição (BARRA et al., 2012, p. 460; SILVEIRA, 2008), fica evidente a importância dada ao diagnóstico das formas "virilizantes simples", ainda que estas não representem um risco à vida dos sujeitos. Os casos "virilizantes simples" que, como visto previamente, podem ter como manifestações clínicas a dita "genitália ambígua", constituem, nesse contexto, um desvio em relação à norma da dicotomia sexual. Desvio que é tratado pelos saberes médicos e psicológicos como uma patologia.

Ainda, segundo a autora, existem outras estratégias diagnósticas que poderiam ser eficazes para reduzir o número de falso-positivos (PEZZUTI, 2010). Todavia, ela aponta para os entraves em relação a esse método, uma vez que ele ainda não teria sido devidamente "comprovado". Silveira (2008, p. 25), por outro lado, argumenta que a triagem para HAC deveria ser realizada apenas em meninos, uma vez que estes não apresentam sinais clínicos, podendo ser diagnosticados clinicamente através da "crise de perda de sal ou da "precocidade sexual' na forma simples". Às meninas, ficaria restrito o diagnóstico clínico, pois essa condição seria facilmente detectada, em função da "virilização genital". Nesse sentido, a autora conclui que "não é consenso que a HAC deva ser incluída em políticas públicas de saúde" (SILVEIRA, 2008, p. 25). Os artigos dão destaque também à ansiedade e ao sofrimento que os resultados falso-positivos podem provocar nas famílias dos sujeitos em vias de diagnóstico pelo programa de triagem, situação em que permanecem até a confirmação ou exclusão diagnóstica de cada criança (PEZZUTI, 2008, p. 68).

A segunda controvérsia diz respeito ao fato dessa condição, em muitos dos casos, não representar um risco à vida (morbimortalidade) ${ }^{11}$ dos indivíduos, um

\footnotetext{
${ }^{11} 0$ conceito de morbimortalidade significa, segundo a definição encontrada no dicionário Aurélio,
} "a relação entre morbidade e mortalidade". A morbidade, por sua vez, diz respeito à incidência de 
dos critérios para inclusão de uma condição nos programas de triagem, pois, conforme mencionado, apenas a forma "perdedora de sal" da HAC coloca a vida do sujeito diretamente em perigo. A "forma virilizante simples" ou a "forma não clássica" não influencia muito a expectativa de vida e, na grande maioria de casos, não representa danos suplementares àqueles que a possuem. Esta forma, ao se constituir como uma ameaça ao modelo de corpo estabelecido culturalmente, traduz-se nos manuais médicos como uma "emergência" no recém-nascido, ainda que o que esteja em jogo seja uma emergência social de "corrigir" a genitália. Assim, sob esta perspectiva, essas crianças são submetidas a uma série de procedimentos cirúrgicos e outras intervenções clínicas invasivas e, muito frequentemente, mutiladoras, desde o período neonatal.

Deste modo, no caso da HAC, salvo os casos de perda de sal, o "registro civil incorreto de meninas" é considerado um fator altamente relevante para inclusão da HAC no programa (CARDOSO et al., 2005, p. 113). Entretanto, no mesmo artigo de Cardoso et al. (2005), conclui-se que a triagem não é adequada para detectar as "formas não clássicas" de HAC, uma vez que quando se estabelece um ponto de corte muito baixo, para detectar todas as formas da doença, há um aumento elevado nos casos falso-positivos. Alguns artigos científicos explicitam que a detecção dos casos "virilizante simples" também é uma prioridade para esse programa de rastreamento. Todavia, tal argumento não coincide com os objetivos estabelecidos pelo Programa Nacional de Triagem Neonatal, conforme previamente mencionado no artigo de Barra et. al (2012).

A terceira controvérsia diz respeito ao atraso no recebimento dos resultados do teste, o qual acaba comprometendo o diagnóstico precoce dos casos com perda de sal que se constitui, conforme descrito nos artigos, como o principal benefício da triagem neonatal pra HAC. Assim, a inserção da HAC na triagem não se justificaria pelo benefício de identificar rapidamente a forma clássica, uma vez que a crise de perda de sal geralmente ocorre nos primeiros dias de vida. Uma questão importante relacionada ao atraso nos resultados do testes, muito mencionada nos textos analisados, é a geração de um alto nível de estresse e sofrimento na família dos bebês em vias de diagnóstico.

Há também aquelas controvérsias relacionadas ao tratamento e às questões bioéticas que acompanham 0 tratamento pré-natal com dexametasona especialmente no que tange à experimentação científica e ao uso de recursos para "prevenir" diferenças corporais que não representam, em grande parte dos casos,

uma doença em uma dada população. Assim, esse conceito sugere a taxa relacional entre a incidência da doença e o número de mortes causada por ela. 
riscos à saúde dos indivíduos. As questões descritas acima também estão presentes no que se refere à terapêutica pós-natal, com o uso de uma série de corticóides que comprometem, de forma substancial, a saúde e o bem-estar dos indivíduos que recebem essas substâncias, as quais acabam gerando complicações, tais como a síndrome de Cushing, a osteoporose e a hipertensão. Tais fatos foram observados tanto em textos médicos, quanto em fóruns de discussão na internet sobre a $\mathrm{HAC}^{12}$.

Alvez et al. (2006, p. 204), em um dos textos analisados, destacam que "também não eram diagnosticados precocemente, antes da implementação desse programa, os recém-nascidos do sexo feminino com acentuada virilização da genitália externa, por serem erroneamente diagnosticados como sendo do sexo masculino". Tal fato demonstra que a criação dessa tecnologia diagnóstica modificou a forma pela qual a "verdade do sexo" (FOUCAULT, 1988) é "revelada" pelos saberes biomédicos, uma vez que, antes de seu surgimento, vários indivíduos, hoje assignados como mulheres e cuja genitália escapa de um determinado padrão, eram designados como homens ao nascimento.

Finalmente, a última das controvérsias diz respeito à colocação da "genitália ambígua" como um "problema de saúde pública", que demanda uma intervenção estatal. Como visto acima, os objetivos previstos pelo programa de triagem não justificariam o diagnóstico das formas de HAC que não representam riscos à saúde dos sujeitos. Contudo, a "virilização" é inserida na categoria de "morbimortalidade" e entendida como uma doença que deve ser tratada e, acima de tudo, prevenida.

\section{APONTAMENTOS FINAIS}

0 objetivo central desse texto consistiu em analisar, através da inclusão da Hiperplasia Adrenal Congênita no Teste do Pezinho, o modo pelo qual a ciência atual, especialmente a partir de aparatos discursivos e tecnológicos da biomedicina, está produzindo os corpos sexuados e as normatizações que recaem sobre eles. Apesar das muitas reivindicações advindas da militância política e/ou dos sujeitos intersexuais não necessariamente envolvidos com 0 ativismo, que denunciam o caráter compulsório e mutilador das intervenções biomédicas, as

\footnotetext{
${ }^{12}$ Embora a análise dos fóruns de discussão online sobre a HAC não esteja contemplada neste artigo, vale destacar que esses trazem informações relevantes aos debates sobre o manejo dessa condição. Tais fóruns são geralmente compostos por pessoas diagnosticadas com HAC, por familiares de sujeitos diagnosticados, e, muitos deles, são também integrados por ativistas.
} 
diferenças corporais e as supostas manifestações clínicas da HAC - que, na grande maioria dos casos, não se traduzem em uma "emergência clínica" (MACHAD0, 2008) - são colocadas no registro da "patologia" e tratados como um problema de saúde pública. Esse projeto, no qual se investe dinheiro público, discursos, tratamentos e recursos diversos, fornece-nos pistas para compreender as dinâmicas de gênero atuais, uma vez que indica quais os corpos e sexualidades são legitimados e desejados neste contexto, tendo em vista que a organização da deteç̧ão de um "desvio" como a HAC não é uma questão apenas "prática”, mas produz "efeitos de realidade", como argumenta Mol (2007).

Ainda, foi possível constatar que, se, por um lado, essa ciência se propõe a desvelar, cada vez com mais "precisão", a realidade dos corpos e, portanto, dos sexos; por outro, acaba, em realidade, "performando" (MOL, 2003) essas materialidades e esses discursos de verdade, através do emprego de novas tecnologias diagnósticas e dos entendimentos subjacentes a esses processos. Neste sentido, principalmente a partir das reflexões de Bruno Latour (2000) sobre controvérsias sociotécnicas, percebeu-se que, embora haja um reconhecimento da multiplicidade dos sexos para além do modelo binário, há também, no processo de produção científica, o esforço de "estabilização" dessas controvérsias. Dito em outros termos, de produção de "caixas pretas" em relação aos corpos, na medida em que seus argumentos e seu manejo acabam reiterando a ideia da existência de apenas dois sexos.

Uma série de questões bioéticas está, também, envolvida, no que diz respeito à questão da autonomia, da experimentação científica e as práticas eugênicas. Estas questões emergiram no que tange, de um modo geral, às intervenções que recaem sobre as pessoas intersexuais, e sobre os corpos das "mães" - no que diz respeito ao uso da dexametasona no período pré-natal -; bem como ao tratamento com substâncias e técnicas que ainda não foram consideradas seguras, devido à falta de estudos longitudinais que avaliem os prejuízos que elas podem causar. Finalmente, pode-se concluir que, retomando a ideia de "variação", trazida por Cabral e Benzur (2005), é necessário pensar a intersexualidade a partir de uma perspectiva despatologizante, nas quais outras corporalidades, que extrapolem o suposto dimorfismo sexual, sejam possíveis. 


\section{REFERÊNCIAS}

BUTLER, Judith. Problemas de gênero: feminismo e subversão da identidade. Rio de Janeiro: Civilização Brasileira, 2003.

CABRAL, Mauro. El cuerpo en el cuerpo: una introdución a las biopolíticas de la intersexualidad. Orientaciones: Revista de Homosexualidades, n.11, Madrid, 2006, p. 47-68.

CABRAL, Mauro. "Presentación"; "Acerca de este libro". In: CABRAL, Mauro (Ed.). Interdicciones. Escrituras de la intersexualidad en castellano. $1^{\mathrm{a}}$ ed. Córdoba: Anarrés Editorial, 2009. p. 5-13.

CABRAL, Mauro; BENZUR, Gabriel. Cuando digo intersex. Um dialogo introductorio a laintersexualidad. Cadernos Pagu, v. 24, Campinas, jun/2005, p. 283-304.

CUNHA, Olívia Maria Gomes da. Tempo imperfeito: uma etnografia do arquivo. Mana, v.10, n.2, Rio de Janeiro, out/ 2004, p. 287-322.

DREGER, Alice Domurat. Hermaphrodites and the medical invention of sex. Cambridge: Harvard University Press. 1998.

DREGER, Alice et al. Prenatal Dexamethasone for Congenital Adrenal Hyperplasia: An Ethics Canary in the Modern Medical Mine, Bioethical Inquiry, v.9, mai/ 2012, p. $277-294$.

DREGER, Alice Domurat; HERNDON, April. Progress and politics in the intersex rights movement. Feminist theory in action. GLQ: A Journal of Lesbian and Gay Studies, v.15, n. 2, Estados Unidos, 2009, p.199-224.

FOUCAULT, Michel. A bistória da sexualidade 1: a vontade de saber. Rio de Janeiro: Edições Graal, 1988.

HARAWAY, Donna. Gênero para um dicionário marxista, Cadernos Pagu, v.22, 2004, p. 201-246.

JESUS, Lisieux Eyer de; BASTOS, Claudio Lyra. Pseudohermafroditismo e estados intersexuais: evolução histórica e impasses do tratamento médico, Psychiatric Online Brasil, v.9, n.5, mai/2004.

KARKAZIS, Katrina. Fixing Sex: Intersex, Medical Authority and Lived Experience. United States: Duke University Press, 2008.

LATOUR, Bruno. Ciência em ação: como seguir cientistas e engenheiros sociedade afora. São Paulo: Editora UNESP, 2000. 
MACHADO, Paula Sandrine. O sexo dos anjos: representações e práticas em torno do gerenciamento sociomédico e cotidiano da intersexualidade. Tese de doutorado, Programa de Pós-graduação em Antropologia Social, Universidade Federal do Rio Grande do Sul, Porto Alegre, RS, 2008.

MOL, Annemarie. Políticas Ontológicas. Algumas idéias e várias perguntas. In: NUNES, João Arriscado e ROQUE, Ricardo (org.) Objectos impuros. Experiências em estudos sociais da ciência. Porto: Edições Afrontamento, 2007.

MOL. Annemarie. The Body Multiple: Ontology in Medical Practice. United States: Duke University Press. 2003.

MONTEIRO, Marko. Dilemas do Humano: reinventando o corpo numa era (bio) tecnológica. Tese de Doutorado. Campinas: Universidade Estadual de Campinas, Instituto de Filosofia e Ciências Humanas. 2005.

ROSE, Nikolas. Biopolítica molecular, ética somática e o espírito do biocapital. In: SANTOS, Luis Henrique Sacchi dos; RIBEIR0, Paula Regina Costa (Orgs.). Corpo, gênero e sexualidade: instâncias e práticas de produção nas políticas da própria vida. Rio Grande: FURG, 2011, p. 13-31.

\section{FONTES PRIMÁRIAS}

ALVEZ, Crésio de Aragão Dantas. et al. Triagem Neonatal para Hiperplasia Adrenal Congênita: Considerações Sobre a Elevação Transitória da 17-hidroxiprogesterona. Revista Brasileira em Promoção da Saúde, v.19, nº 4, Fortaleza, 2006, p. 203-208.

BACHEGA, Tânia et al. Tratamento da hiperplasia supra-renal congênita por deficiência da 21-hidroxilase. Arquivos Brasileiros de Endocrinologia e Metabologia, São Paulo, v. 45, nº 1, fev/ 2001, p. 64-72.

BARRA, Cristina Botelho Barra et al. Triagem neonatal para hiperplasia adrenal congênita. Revista Associação Médica Brasileira. v. 58, nº 4, jul/ ago/2012, São Paulo, p.459-464.

BRASIL. Ministério da Saúde. Secretaria de Assistência à Saúde. Coordenação-Geral de Atenção Especializada. Manual de Normas Técnicas e Rotinas Operacionais do Programa Nacional de Triagem Neonatal. Brasília: Ministério da Saúde, 2002.

BRASIL, Ministério da Saúde. Portaria n ${ }^{0} 2.829$ de 14 de Dezembro de 2012. Inclui a Fase IV no Programa Nacional de Triagem Neonatal (PNTN), instituído pela Portaria $n^{\circ} 822 / G M / M S$, de 6 de junbo de 2001. Ministério da Saúde. Disponível em: 
<http://bvsms.saude.gov.br/bvs/saudelegis/gm/2012/prt2829_14_12_2012.html $>$. Acesso em: 14 jun. 2014.

CARDOSO, Cláudia B.M.A. et al . Triagem neonatal para hiperplasia adrenal congênita: experiência do estado do Rio de Janeiro. Arquivos Brasileiros de Endocrinologia e Metabologia, v. 49, n. 1, Fev/2005, São Paulo, p.112-119.

ESTADO DO RIO GRANDE DO SUL, Secretária da Saúde. Resolução nº 591 de 11 de Novembro de 2013. Repassa a Secretaria Municipal de Saúde de Porto Alegre verba para adaptação do Serviço de Referência em Triagem Neonatal e do Laboratório em Triagem Neonatal do Hospital Materno Infantil Presidente Vargas e montagem de rede de Urgência para os casos de Hiperplasia Adrenal Congênita. Secretaria da Saúde. Disponível em:

$<$ http://www.saude.rs.gov.br/upload/1385039578_cibr591_13.pdf>. Acesso em 20 jun.2014.

PEZZUTI, Isabela Leite. Avaliação do programa-piloto de triagem neonatal para hiperplasia adrenal congênita no estado de Minas Gerais. Dissertação de Mestrado, Ciências da Saúde, Universidade Federal de Minas Gerais, Belo Horizonte, 2010.

PEZZUTI Isabela Leite et al. A three-year follow-up of congenital adrenal hyperplasia newborn screening. Jornal de Pediatria, v.90, n.3 mai/jun/ 2014, Rio de Janeiro, p.300-307.

HAYASHI, Giselle. et al. Weight-adjusted neonatal 170H-progesterone cutoff levels improve the efficiency of newborn screening for congenital adrenal hyperplasia. Arquivos Brasileiros de Endocrinologia e Metabologia, v.55, n. 8, 2011, São Paulo, p. 632-637.

SILVEIRA, Elizabeth Lemos. Hiperplasia adrenal congênita no Brasil: incidência, custos da triagem neonatal e aplicação clínica da biologia molecular. Tese de doutorado, Ciências Médicas: Endocrinologia. Porto Alegre, Universidade Federal do Rio Grande do Sul, 2008.

SOARDI. Fernanda Caroline. et al. Heterozygosis for CYP21A2 Mutation Considered as 21-Hydroxylase Deficiency in Neonatal Screening. Arquivos Brasileiros de Endocrinologia e Metabologia, v.52, n. 8 , 2008, São Paulo, p. 1388-92.

YARAK, Samira, et al. Hiperandrogenismo e pele: síndrome do ovário policístico e resistência periférica à insulina. Anais Brasileiros de Dermatologia, v.80, n.4, 2005, p.395-410. 